\title{
Safety and Efficacy of Endoscopic Mucosal Therapy with Radiofrequency Ablation for Patients with Neoplastic Barrett's Esophagus
}

\author{
William J. Bulsiewicz, MD, MSc, Hannah P. Kim, BA, Evan S. Dellon, MD, MPH, Cary C. \\ Cotton, BA, Sarina Pasricha, MD, Ryan D. Madanick, MD, Melissa B. Spacek, FNP, Susan E. \\ Bream, BS, Xiaoxin Chen, MD, PhD, Roy C. Orlando, MD, and Nicholas J. Shaheen, MD, \\ MPH \\ Center for Esophageal Diseases and Swallowing, Department of Medicine, Division of \\ Gastroenterology, University of North Carolina School of Medicine, Chapel Hill, NC
}

\begin{abstract}
Background\& Aims-The goal of radiofrequency ablation (RFA) for patients with Barrett's esophagus (BE) is to eliminate dysplasia and metaplasia. The efficacy and safety of RFA for patients with BE and neoplasia are incompletely characterized.

Methods-We performed a retrospective study of 244 patients treated with RFA for BE with dysplasia or intramucosal carcinoma. Efficacy outcomes were complete eradication of intestinal metaplasia (CEIM), complete eradication of dysplasia (CED), total treatments, and RFA sessions. Safety outcomes included death, perforation, stricture, bleeding, and hospitalization. We identified factors associated with incomplete EIM and stricture formation.
\end{abstract}

Results-CEIM was achieved in 80\% of the patients, and CED in 87\%; disease progressed in 4 patients. A higher percentage of patients with incomplete EIM were female (40\%) than those with CEIM (20\%, $P=.045$ ); patients with incomplete EIM also had a longer segment of BE (5.5 vs 4.0 $\mathrm{cm}, P=.03$ ), incomplete healing between treatment sessions ( $45 \%$ vs $15 \%, P=0.004)$, and underwent more treatment sessions ( 4 vs $3, P=.007$ ). Incomplete healing was independently associated with incomplete EIM. Twenty-three patients $(9.4 \%)$ had a treatment-related complication during 777 treatment sessions (3.0\%), including strictures (8.2\%), post-procedural hemorrhages (1.6\%), and hospitalizations (1.6\%). Patients that developed strictures were more likely to use non-steroidal anti-inflammatory drugs (NSAID) than those without strictures (70\% vs $45 \%, P=.04)$, have undergone antireflux surgery ( $15 \%$ vs $3 \%, P=.04)$, or had erosive esophagitis (35\% vs $12 \%, P=.01)$.

(C) 2012 The American Gastroenterological Association. Published by Elsevier Inc. All rights reserved.

Correspondence: Nicholas J. Shaheen, MD, MPH, Professor of Medicine and Epidemiology, Director, Center for Esophageal Diseases and, Swallowing, University of North Carolina School of Medicine, CB\#7080, Chapel Hill, NC 27599-7080.

Authors Contributions: conception and design - WB, ED, RM, MS, XC, RO, NS; analysis and interpretation of the data - WB, HK, CC, ED, SP, NS; article drafting- WB, HK, ED, NS; all authors approved final draft Writing Assistance: Not applicable.

Conflict of Interest Disclosure: Dr. Shaheen reports research grants from BARRX Medical, Oncoscope, CSA Medical, Takeda, and Astra Zeneca. He is a consultant for CSA Medical, Astra Zeneca, Takeda, Oncoscope, and Neo Genomics. Dr. Dellon reports a research grant from Astra Zeneca and is a consultant for Oncoscope.

The remaining authors have no conflicts of interest.

Publisher's Disclaimer: This is a PDF file of an unedited manuscript that has been accepted for publication. As a service to our customers we are providing this early version of the manuscript. The manuscript will undergo copyediting, typesetting, and review of the resulting proof before it is published in its final citable form. Please note that during the production process errors may be discovered which could affect the content, and all legal disclaimers that apply to the journal pertain. 
Conclusions-RFA is highly effective and safe for treatment of BE with dysplasia or earlystage cancer. Strictures were the most common complications. Incomplete healing between treatment sessions was associated with incomplete EIM. NSAID use, prior anti-reflux surgery, and a history of erosive esophagitis predicted stricture formation.

\section{Keywords}

Barrett's esophagus; radiofrequency ablation; esophageal cancer; epidemiology

\section{Introduction}

The goal of endoscopic eradication therapy for Barrett's esophagus (BE) is to eliminate dysplasia and intestinal metaplasia to prevent neoplastic progression to esophageal adenocarcinoma. Recent guidelines recommend endoscopic eradication therapy with radiofrequency ablation (RFA) and/or endoscopic mucosal resection (EMR) for treatment of patients with BE and high-grade dysplasia (HGD). ${ }^{1}$ Further, "RFA should also be a therapeutic option for treatment of patients with confirmed low-grade dysplasia (LGD) in Barrett's esophagus."1

Although there is no consensus on whether all subjects with LGD should be treated with RFA, the guidelines advocate for shared decision making based upon a discussion of risks and benefits between the physician and patient. Despite the emerging role of RFA in the treatment of neoplastic BE (LGD, HGD, and intramucosal carcinoma [IMC]), limited data are available regarding the safety and efficacy of RFA in this cohort. No reports detail the efficacy of RFA for treatment of IMC. Additionally, risk factors for incomplete eradication of intestinal metaplasia, as well as risk factors for stricture formation following treatment, are poorly understood.

We performed a retrospective study to assess the safety and efficacy of RFA for the treatment of neoplastic Barrett's esophagus in 244 patients: 53 with LGD, 152 with HGD, and 39 with IMC. Additionally, we analyzed factors associated with complete eradication of intestinal metaplasia (CEIM) and stricture formation.

\section{Methods}

\section{Data collection and patient eligibility}

We performed a retrospective study of consecutive patients with $\mathrm{BE}$ and confirmed neoplasia (LGD, HGD, IMC) treated with RFA at University of North Carolina (UNC) Hospitals between 2006 and 2011. To identify all such subjects at our institution, we searched our electronic endoscopic database (ProvationMD, Wolters Kluwer, Minneapolis, MN) from January 1, 2006 through November 1, 2011 using the following terms: Barrett, esophageal adenocarcinoma, cancer, carcinoma in situ, dysplasia, ablation, radiofrequency. We also searched for procedure codes foresophagoscopy with ablation (CPT 43228).

Each subject was reviewed by one of two investigators (WB, HK) using the electronic medical record (WebCIS, University of North Carolina Health Care System) to determine eligibility for inclusion. All institutional health information plus imported external records were reviewed. Subjects were excluded if they never had treatment with RFA, were treated with RFA for a non-BE related disease, or did not have neoplastic BE. All subjects with neoplastic BE treated with RFA were studied for safety outcomes (safety cohort). The efficacy analysis was restricted to exclude patients receiving ongoing RFA therapy as of November 1, 2011. 
Pertinent data were extracted from clinical, endoscopy, and pathology reports for each subject, including: demographic information (age, gender, race, body mass index), medical and social history (erosive esophagitis, peptic stricture, alcohol/tobacco use), medication use (antisecretory therapy, non-steroidal anti-inflammatory use), pre-treatment histology, endoscopy findings (Prague $\mathrm{C}$ and $\mathrm{M}$ class, presence of ulcer, esophagitis or nodule), treatment provided, ablation outcomes, and complications. To standardize methodology, the first ten subjects were reviewed by both investigators jointly with discrepancies in data collection resolved by consensus. For additional quality control, every $20^{\text {th }}$ study subject was reviewed independently by both investigators to assess inter-rater agreement.

\section{Pre-treatment evaluation and procedural protocol}

All patients had an initial consultation visit to discuss BE and dysplasia, its risk of progression to cancer, and the risks and benefits of various treatment strategies. Prior to the visit, the worst histologic grade of BE was determined by review of original pathology reports. In all cases, review of pathology slides by a second expert gastrointestinal pathologist was performed as part of the patient's routine care, and if findings were discordant, by an additional expert gastrointestinal pathologist for categorization.

Patients with BE and LGD were offered RFA plus endoscopic surveillance or endoscopic surveillance alone. Patients with BE and HGD or IMC were offered RFA plus endoscopic surveillance, esophagectomy, or endoscopic surveillance alone. Cases with lymphatic or vasoinvasive tumor growth or poor tumor differentiation were not considered for curative RFA therapy. Patients with HGD or IMC who elected for RFA had pre-treatment staging by upper endoscopy (EGD) and endoscopic ultrasound (EUS) to identify findings of advanced disease that would preclude curative endoscopic treatment (submucosal infiltration, lymph node or metastatic spread). Patients with nodular disease had endoscopic mucosal resection (EMR) using either the Olympus $18 \mathrm{~mm}$ oblique cap kit (Olympus America, Center Valley, PA), or the Duette device (Cook Medical, Winston-Salem, NC). All EMRs performed with the Olympus system were performed after submucosal injection of saline, while Duette EMR was performed without prior injection. Any residual nodularity due to lateral extension of the IMC was excised at subsequent examination. EMR of non-nodular mucosa was not performed. RFA was performed 2 months after the final EMR, provided the pathology specimen did not have submucosal involvement of carcinoma, and that the mucosa after EMR demonstrated no residual nodularity. Twice daily proton pump inhibitor therapy was prescribed prior to and throughout the endoscopic therapy period.

Radiofrequency ablation was performed using the $\mathrm{HALO}^{360}$ (circumferential) and $\mathrm{HALO}^{90}$ (focal) devices developed by BÂRRX Medical (Sunnyvale, CA). Standard procedural technique was used. ${ }^{2}$ After primary circumferential ablation, patients had repeat upper endoscopy every 2 months to assess response to treatment. Nodular BE was treated with EMR. Residual, non-nodular BE was treated with focal ablation. Circumferential treatment of the Z-line was performed using the HALO ${ }^{90}$ device as a part of all focal treatment sessions. Patients with visible breaks in the esophageal mucosa during treatment were considered to have incomplete healing between treatment sessions, and were treated with intensification of acid suppressive therapy. If no visible BE was present on white-light endoscopy and narrow-band imaging, four-quadrant biopsies were performed in the tubular esophagus with large capacity forceps every $1 \mathrm{~cm}$ spanning the entire original length of the $\mathrm{BE}$ segment and extending to the top of the gastric folds. If these biopsies demonstrated no persistent BE, treatment was considered complete, and the patient entered into endoscopic surveillance. Treatment could be terminated prior to complete eradication of intestinal metaplasia (CEIM), either at the request of the patient, or if, in the physician's judgment, no further progress was made in inducing neosquamous epithelium. 


\section{Outcomes and statistical analysis}

All biopsy specimens were reviewed by an expert gastrointestinal pathologist to determine the presence of residual BE and degree of dysplasia. Efficacy outcomes included complete eradication of intestinal metaplasia (CEIM), complete eradication of dysplasia (CED), RFA treatment sessions, and total treatment sessions. CEIM was defined as complete endoscopic resolution on white-light endoscopy and narrow-band imaging, as well as the absence of intestinal metaplasia from all histologic specimens. CED was defined as the absence of dysplasia from all histologic specimens. Total treatment sessions include any ablation or endoscopic resection treatment. Primary safety outcomes included deaths, perforations, strictures, bleeding, and hospitalizations. A stricture was defined as any narrowing of the esophageal lumen requiring dilation. Bleeding was considered clinically significant if it required hospitalization or a blood transfusion.

Efficacy outcomes are reported per protocol (PP) and on an intention-to-treat (ITT) basis. Patients who were lost to follow-up, were diagnosed with an unrelated life-threatening comorbidity that precluded further treatment, or died of an unrelated event during the treatment period were included as treatment failures for the ITT analysis, but were excluded from the PP analysis.

Patient characteristics associated with incomplete elimination of intestinal metaplasia and stricture formation were assessed by comparing patients with these outcomes to those without using non-parametric statistics (Fisher's exact test for categorical variables, Wilcoxon rank sum test for continuous variables). Bivariable comparisons were made for age, gender, body mass index, pre-treatment histology, NSAID use, medium or larger hiatus hernia, length of Barrett's segment, presence of nodular disease, EMR during treatment, incomplete healing between treatment sessions, and number of treatment sessions. The characteristics most strongly associated with incomplete elimination of intestinal metaplasia and stricture formation were included in a multivariable logistic regression model to determine independent predictors of these outcomes. Given the limited number of patients with incomplete elimination of intestinal metaplasia and stricture, we restricted our multivariable model to avoid overfitting.

Among patients that achieved complete elimination of intestinal metaplasia, we plotted the number of RFA treatment sessions to CEIM. Characteristics independently predictive of the number of RFA sessions to eliminate intestinal metaplasia were assessed in a linear regression model. Each of the characteristics assessed in the bivariable comparisons described above were included in this model. Statistical significance was defined as a pvalue less than 0.05 .

\section{Results}

\section{Patient Eligibility}

A total of 286 patients received EGD with ablative therapy at UNC Hospitals between 2006 and 2011. Of these patients, 42 were excluded: 19 never received RFA and were treated with a different ablative modality (15 cryoablation, 2 argon plasma coagulation, 2 photodynamic therapy); 5 were treated for a non-BE related disease ( 3 squamous cell carcinoma, 1 gastric antral vascular ectasia, 1 gastric cardia dysplasia); and 18 had pre-ablation histology other than LGD, HGD or IMC (11 nondysplastic BE, 7 invasive adenocarcinoma). The remaining 244 patients with LGD, HGD or IMC treated with RFA at UNC were included in the safety analysis.

An additional 34 patients were receiving ongoing RFA therapy as of November 1, 2011 and were excluded from the efficacy analysis. The remaining 210 patients were included in the 
intention-to-treat efficacy analysis. Within this cohort, 22 patients did not complete treatment and were considered treatment failures: of these 22, 14 were lost to follow-up, 6 were diagnosed with an unrelated life-threatening comorbidity that halted further treatment, and 2 died of an unrelated event during the treatment period. These 22 patients were excluded from the per protocol analysis. The 14 subjects lost to follow-up did not differ significantly from the remainder of the cohort with respect to pre-treatment histology or median number of treatment sessions.

\section{RFA Efficacy and Characteristics Associated with CEIM}

Among 210 patients analyzed for efficacy (ITT), there were 44 with LGD, 135 with HGD, and 31 with IMC. Most patients were Caucasian men, and on average, patients were 64 years old with a BMI of $29.7 \mathrm{~kg} / \mathrm{m}^{2}$. Baseline characteristics of these patients are summarized in Table 1 .

Overall, patients with dysplastic BE or early cancer had CED in 87\% (97\% PP) and CEIM in $80 \%$ (89\% PP; Table 2). Complete treatment of BE required $3.3 \pm 1.8$ total treatment sessions on average, of which $2.8 \pm 1.7$ were RFA treatment sessions (the remainder represented EMR sessions). Circumferential ablation was performed in 126 of 210 patients (60\%), with 120 of these 126 patients receiving just one circumferential treatment. EMR was performed in 69 of 210 patients (33\%), with 58 of 69 having one endoscopic resection. Four patients experienced disease progression during the study period despite RFA treatment. Three patients with pre-ablation HGD progressed to IMC $(n=1)$ and EAC $(n=2)$, and underwent successful treatment with esophagectomy. The fourth patient had preablation IMC that progressed to EAC. He underwent chemotherapy and radiation, and subsequently opted for esophagectomy for a proximal stage Ia lesion and a distal stage IIb lesion.

RFA was effective at eliminating intestinal metaplasia and dysplasia for all histologic grades of dysplastic BE and early cancer. CED was achieved in 93\% with LGD (100\% PP), 84\% with HGD (97\% PP), and 87\% with IMC (93\% PP). CEIM was achieved in $86 \%$ with LGD (93\% PP), $79 \%$ with HGD (90\% PP), and 77\% with IMC ( $83 \%$ PP). LGD and HGD required approximately 3 total treatmentsessions to complete therapy (LGD $3.2 \pm 1.9$, HGD $3.1 \pm 1.6$ ) while IMC required an average of $4.1 \pm 2.4$ total treatment sessions to complete therapy. The difference in total treatment sessions between IMC and dysplastic BE was due to the increased number of EMRs necessary in the IMC group; EMR was performed at least once in $87 \%$ of patients with IMC (27/31) compared to $28 \%$ of patients with HGD (38/135) and $9 \%$ of patients with LGD (4/44). The total number of RFA sessions to complete therapy did not differ by pre-treatment histology (LGD $3.0 \pm 2.0$, HGD $2.7 \pm 1.5$, IMC $2.9 \pm 2.0$ ).

Intestinal metaplasia was not completely eradicated in 20 of $188(10.6 \%)$ patients in the per protocol analysis (Table 3). Compared to patients who achieved CEIM, patients with incomplete eradication of BE were: 1) more likely to be female ( $40 \%$ vs $20 \%, \mathrm{p}=0.045$ ); 2 ) had greater length of BE ( 5.5 vs. $4.0 \mathrm{~cm}, \mathrm{p}=0.03) ; 3$ ) were more likely to have incomplete healing between treatment sessions ( $45 \%$ vs $15 \%, \mathrm{p}=0.004)$; and 4 ) required more total treatment sessions to complete therapy (median 4.0 vs $3.0, \mathrm{p}=0.007$ ). Interestingly, pretreatment histology was not a predictor of incomplete EIM. Although rates of CEIM did differ numerically by pre-treatment histology (Table 2), these differences did not reach statistical significance ( $\mathrm{p}$ for trend $=0.20$ ). Also, age, body mass index, or the presence of nodular BE at baseline were not significantly associated with achieving CEIM. Incomplete healing between treatment sessions, length of $\mathrm{BE}$ and number of treatment sessions were most strongly associated with incomplete EIM on bivariable analysis. Therefore, these three variables were included in our logistic regression analysis. After performing multivariate analysis, incomplete healing between treatment sessions (OR 3.7; 95\% CI 1.3-9.9) was an 
independent predictor of incomplete EIM after controlling for length of BE and total number of treatment sessions to complete therapy.

CEIM was achieved in more than half of patients after 3 RFA sessions and $75 \%$ after 4 sessions (Figure 1). The number of RFA sessions to achieve CEIM was independently associated with the length of the Barrett's segment ( $\mathrm{p}<0.001)$. The predicted number of treatment sessions for patients with 2-, 5-, and 8cm of Barrett's was 2.1, 2.7 and 3.4, respectively (Figure 2). On average, about one additional treatment session was necessary for each $5 \mathrm{~cm}$ of Barrett's. Patients with nodular disease required 0.8 less RFA sessions $(\mathrm{p}=0.02)$, although the total treatment sessions did not differ due to the need for EMR in these patients. Patients with incomplete healing between treatment sessions required 0.6 more RFA sessions ( $\mathrm{p}=0.02$ ). Age, gender, BMI, NSAID use, hernia size, and pre-treatment histology were not associated with the number of sessions to achieve CEIM.

\section{RFA Safety and Characteristics Associated with Stricture}

Among 244 patients analyzed for safety, there were 53 with LGD, 152 with HGD, and 39 with IMC. On average, patients were 65 years old, most were Caucasian (6 AfricanAmerican), most were men (192/244, 79\%), and the average body mass index was $29.6 \mathrm{~kg} /$ $\mathrm{m}^{2}$. Patients had a median length of BE of $4 \mathrm{~cm}$ (IQR: $2-7$ ) and $30 \%$ had nodular BE at baseline (73/244). Overall, 110 EMR treatments and 667 RFA treatments (158 circumferential, 509 focal) were administered ( 777 total treatment sessions). There were 94 patients treated with EMR (39\%), of which 78 had one EMR treatment (83\%), and 16 had two treatments. There were 146 patients treated with circumferential ablation $(60 \%)$, of which 139 had one circumferential RFA treatment (95\%). Patients had an average of $3.2 \pm$ 1.9 total treatment sessions, including $2.7 \pm 1.7$ RFA treatment sessions.

Complications occurred in 24 of 244 patients (9.8\% of patients, $3.1 \%$ of procedures) (Table 4). There were no deaths or esophageal perforations. Most complications were strictures, which occurred in 20 patients ( $8.2 \%$ of patients, $2.6 \%$ of procedures) after a median of 63 days after initial treatment. Two of these strictures occurred after EMR, and the other 18 after RFA. All but one of the strictures were detected on routine follow-up endoscopy and most were free of dysphagia. Stricture rates did not different significantly based upon histology, occurring in: 6/53 with LGD (11.3\%), 9/152 with HGD (5.9\%) and 5/39 with IMC (12.8\%). All strictures were $<1 \mathrm{~cm}$ in length, and resolved after a median of 1 endoscopic dilation (maximum of 4 dilations). Four of the 20 patients (20\%) had stricture recurrence noted on a subsequent endoscopy, and 3 of these patients required an additional endoscopic dilation. Post-procedural hemorrhage occurred in four patients ( $1.6 \%$ of patients, $0.5 \%$ of procedures), with 2 occurring after EMR and 2 occurring after RFA. In each case, the bleeding was noted during the endoscopy and treated with injection of epinephrine at the bleeding site plus either hemostatic clips ( 3 of 4 ) or bipolar cauterization ( 1 of 4 ). All four of these patients were subsequently hospitalized, with 1 patient receiving transfusion. Three of these four were discharged the next day, and the last had a hospitalization of 2 days. There were no other hospitalizations.

The characteristics of patients with and without stricture during treatment are listed in Table 4. Compared to patients who did not develop a stricture, patients who developed a stricture were more likely to: 1 ) use NSAIDs (70\% vs. $45 \%, \mathrm{p}=0.04) ; 2$ ) have had antireflux surgery before treatment ( $15 \%$ vs. $3 \%, \mathrm{p}=0.04)$; and 3 ) have a history of prior erosive esophagitis (35\% vs. $12 \%, \mathrm{p}=0.01)$. Although not statistically significant ( $>0.05)$, patients with stricture were more frequently female (35\% vs. $20 \%$ ), had incomplete healing between treatment sessions (35\% vs. 17\%), and had an EMR after RFA treatment initiation (20\% vs. $9 \%)$. Stricture was not statistically associated with pre-treatment histology, although patients with stricture were numerically more likely to have IMC before treatment (25\%) compared 
to those without stricture (15\%). The three variables associated with stricture formation on bivariable analaysis were included in our multivariate analysis. NSAID use (OR 3.1; 95\% CI 1.1-8.7), prior antireflux surgery (OR 6.6; 95\% CI 1.5-29.6), and a history of prior erosive esophagitis (OR 3.7; 95\% CI 1.3-10.5) were all independent predictors of stricture formation.

\section{Discussion}

The outcomes of treatment of neoplastic BE with RFA are not well-described, nor are risk factors for complications or incomplete eradication. This study reports our experience treating 244 patients with neoplastic BE using RFA. We found that RFA was highly effective at eradicating dysplasia and intestinal metaplasia, with at least 77\% CEIM (83\% $\mathrm{PP})$ and $84 \%$ CED (93\% PP) regardless of degree of dysplasia or the presence of IMC. Male gender, shorter length of $\mathrm{BE}$, and complete healing of the mucosa between treatment sessions were associated with CEIM, with complete healing of the mucosa being an independent predictor of CEIM. Length of the Barrett's segment, presence of nodularity, and incomplete healing independently predicted the number of RFA treatments to achieve CEIM. Complications occurred in roughly $10 \%$ of patients, with most being benign strictures that were amenable to endoscopic dilation. History of prior erosive esophagitis, NSAID use, and previous Nissen fundoplication were independent predictors of stricture formation.

Published reports of the efficacy and safety of RFA for neoplastic BE are heterogeneous. Reported rates of complete eradication of intestinal metaplasia (CEIM) vary from 54-100\% for HGD and $71-100 \%$ for LGD. ${ }^{3}$ Similarly, published rates of stricture and bleeding vary from $0-8 \%$ and $0-10 \%$, respectively. ${ }^{3}$ Prior to the present study, the largest reported cohort of patients treated with RFA for neoplastic BE was an early report of a multicenter registry of 142 patients with HGD enrolled at 16 institutions. ${ }^{4}$ In contrast to the present study, CEIM was achieved in just $54 \%$ of patients in that study; however patients received only circumferential RFA, as the focal RFA device was not yet developed. In the randomized, sham-controlled AIM Dysplasia Trial, 42 patients with LGD and 42 patients with HGD received up to 4 RFA treatment sessions over the course of 9 months. Biopsies taken at 12 months showed CEIM in $74 \%$ with HGD and $81 \%$ with LGD, response rates slightly lower than the current report. ${ }^{5}$ Of note, this study restricted the number of treatments to four, and a subsequent report noted that further treatment resulted in additional cases of CEIM. ${ }^{6}$

Several findings of our study merit mention. This study identified incomplete mucosal healing between treatment sessions as an independent predictor of incomplete eradication of intestinal metaplasia. Incomplete healing may be a marker of incompletely controlled reflux disease, despite the high dose (twice daily) proton pump inhibitor therapy used in thispatient population. Previous work has demonstrated that a substantial minority of subjects with BE continue to experience pathological reflux, even with chronic high dose PPI therapy. 7,8 Although this explanation seems tenable, the lack of routine $\mathrm{pH}$ testing in this population in the current study makes us unable to comment on the potential role of inadequate acid suppression in subjects with incomplete eradication in this study.

Other findings of note were that combined treatment with EMR and RFA was effective for the treatment of IMC (77\% CEIM, 87\% CED) in the 31 patients reported here. Previous data from smaller series did not assess the efficacy of RFA specifically for subjects who had developed IMC. ${ }^{9}$ Of note, 24 of 31 patients with IMC had nodular disease and were treated with pre-RFA EMR, excising all nodular IMC prior to treatment with RFA. Also, our study assessed the risk factors for stricture development with therapy. Previous studies attempting to do so had inadequate power to identify any predictors, due to small sample size and the 
limited number of patients with stricture formation. ${ }^{10}$ Interestingly, in the present study, NSAID use was associated with an increased likelihood of stricture formation. Some clinicians might consider routine administration of NSAIDs or aspirin to BE patients due to the large body of epidemiological data suggesting a protective effect of NSAIDs and aspirin on the development of esophageal cancer. ${ }^{11}$ These data suggest that this practice might not be advisable during attempts at endoscopic ablation, perhaps due to direct mucosal injury from these medicines.

There are several strengths to our study. This study represents the largest published cohort of patients with neoplastic BE treated with RFA. Biopsy samples were analyzed by expert gastrointestinal pathologists, with all dysplasia confirmed by 2 readings. Our study definitions were conservative, counting any subject as a treatment failure in intention-totreat analysis if therapy was halted for any reason prior to eradication of dysplasia or intestinal metaplasia. Our study also had several limitations. One limitation is that this study was conducted at a tertiary-care referral center. Endoscopies and interventions were performed by the same experienced gastroenterologists. Therefore, whether or not these results can be generalized to community practice settings is unknown. The limited number of incomplete responders in this study, as well as our relatively small numbers of complications, may have limited our ability to detect independent predictors of incomplete eradication or stricture formation in our multivariate analysis. For example, longer segment length seems intuitive as a proxy for burden of disease, and has been suggested as a predictor of incomplete response in previous work. ${ }^{12}$ Finally, despite our rigorous attempts at complete collection of data, the retrospective nature of this study makes underestimation of complications or misclassification errors possible.

In summary, RFA is a safe and effective treatment option for patients with BE with LGD, HGD or IMC. CEIM was achieved in at least $77 \%$ of patients regardless of degree of dysplasia. Patients with complete healing of their mucosa between treatment sessions are more likely to have CEIM and length of the Barrett's segment predicts the number of RFA treatments that will be required. Complications occurred in around 10\% of patients, most of which were benign strictures that were amenable to endoscopic dilation. Patients who used NSAIDs, have a history of prior erosive esophagitis, or those who had a previous surgical antireflux procedure were more likely to have a stricture. The findings of this study can be used to inform treatment decisions and appropriately counsel patients with neoplastic BE being considered for treatment with RFA.

\section{Acknowledgments}

Grant Support: This research was supported, in part, by award number T32 DK07634 from the National Institutes of Health, award number KL2RR025746 from the National Center for Research Resources, and a grant from the Doris Duke Charitable Foundation.

\section{Abbreviations}

$\begin{array}{ll}\text { BE } & \text { Barrett's esophagus } \\ \text { CED } & \text { complete eradication of dysplasia } \\ \text { CEIM } & \text { complete eradication of intestinal metaplasia } \\ \text { EMR } & \text { endoscopic mucosal resection } \\ \text { EUS } & \text { endoscopic ultrasound } \\ \text { HGD } & \text { high-grade dysplasia }\end{array}$




$\begin{array}{ll}\text { IMC } & \text { intramucosal carcinoma } \\ \text { ITT } & \text { intention-to-treat } \\ \text { LGD } & \text { low-grade dysplasia } \\ \text { NSAID } & \text { nonsteroidal anti-inflammatory drug } \\ \text { PP } & \text { per protocol } \\ \text { RFA } & \text { radiofrequency ablation } \\ \text { UNC } & \text { University of North Carolina }\end{array}$

\section{References}

1. Spechler SJ, Sharma P, Souza RF, et al. American Gastroenterological Association medical position statement on the management of Barrett's esophagus. Gastroenterology. 2011; 140:1084-91. [PubMed: 21376940]

2. Sharma VK, Jae Kim H, Das A, et al. Circumferential and focal ablation of Barrett's esophagus containing dysplasia. Am J Gastroenterol. 2009; 104:310-7. [PubMed: 19174783]

3. Bulsiewicz WJ, Shaheen NJ. The role of radiofrequency ablation in the management of Barrett's esophagus. Gastrointest Endosc Clin N Am. 2011; 21:95-109. [PubMed: 21112500]

4. Ganz RA, Overholt BF, Sharma VK, et al. Circumferential ablation of Barrett's esophagus that contains high-grade dysplasia: a U.S. Multicenter Registry. Gastrointest Endosc. 2008; 68:35-40. [PubMed: 18355819]

5. Shaheen NJ, Sharma P, Overholt BF, et al. Radiofrequency ablation in Barrett's esophagus with dysplasia. N Engl J Med. 2009; 360:2277-88. [PubMed: 19474425]

6. Shaheen NJ, Overholt BF, Sampliner RE, et al. Durability of radiofrequency ablation in Barrett's esophagus with dysplasia. Gastroenterology. 2011; 141:460-8. [PubMed: 21679712]

7. Wani S, Sampliner RE, Weston AP, et al. Lack of predictors of normalization of oesophageal acid exposure in Barrett's oesophagus. Aliment Pharmacol Ther. 2005; 22:627-33. [PubMed: 16181302]

8. Gerson LB, Shetler K, Triadafilopoulos G. Control of intra-oesophageal and intra-gastric pH with proton pump inhibitors in patients with Barrett's oesophagus. Dig Liver Dis. 2005; 37:651-8. [PubMed: 15919250]

9. Pouw RE, Wirths K, Eisendrath P, et al. Efficacy of radiofrequency ablation combined with endoscopic resection for barrett's esophagus with early neoplasia. Clin Gastroenterol Hepatol. 2010; 8:23-9. [PubMed: 19602454]

10. Okoro NI, Tomizawa Y, Dunagan KT, et al. Safety of prior endoscopic mucosal resection in patients receiving radiofrequency ablation of Barrett's esophagus. Clin Gastroenterol Hepatol. 2012; 10:150-4. [PubMed: 22056303]

11. Corley DA, Kerlikowske K, Verma R, et al. Protective association of aspirin/NSAIDs and esophageal cancer: a systematic review and meta-analysis. Gastroenterology. 2003; 124:47-56. [PubMed: 12512029]

12. Korst RJ, Santana-Joseph S, Rutledge JR, et al. Effect of hiatal hernia size and columnar segment length on the success of radiofrequency ablation for Barrett's esophagus: a single-center, phase II clinical trial. J Thorac Cardiovasc Surg. 2011; 142:1168-73. [PubMed: 21840549] 


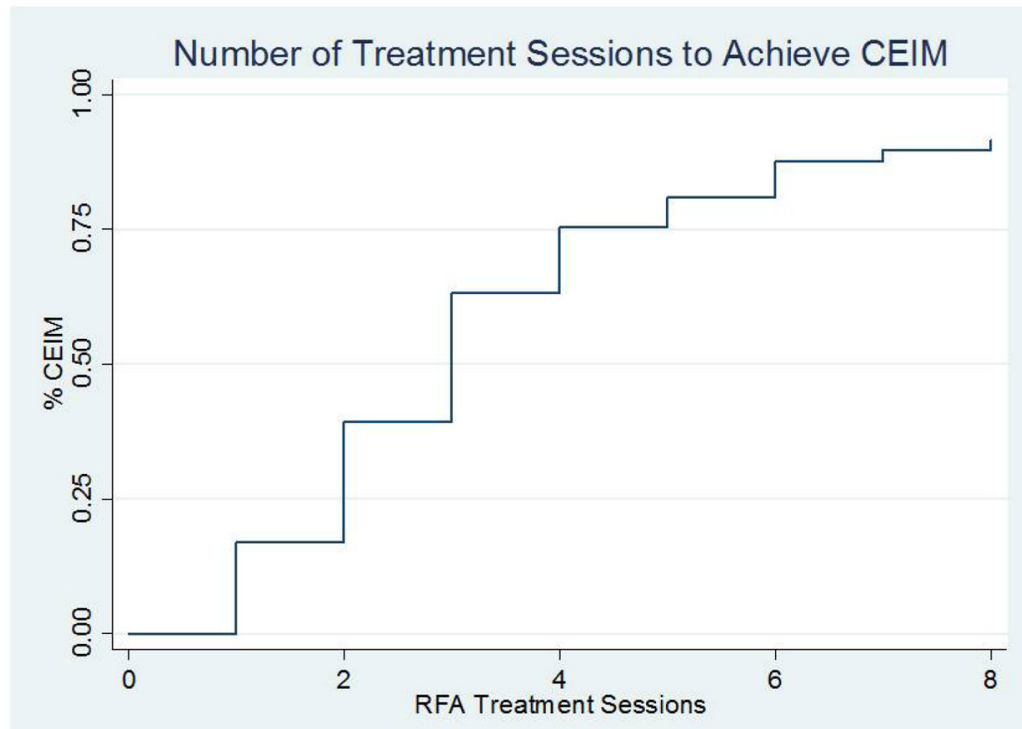

Figure 1.

Number of treatment sessions to eliminate intestinal metaplasia. 


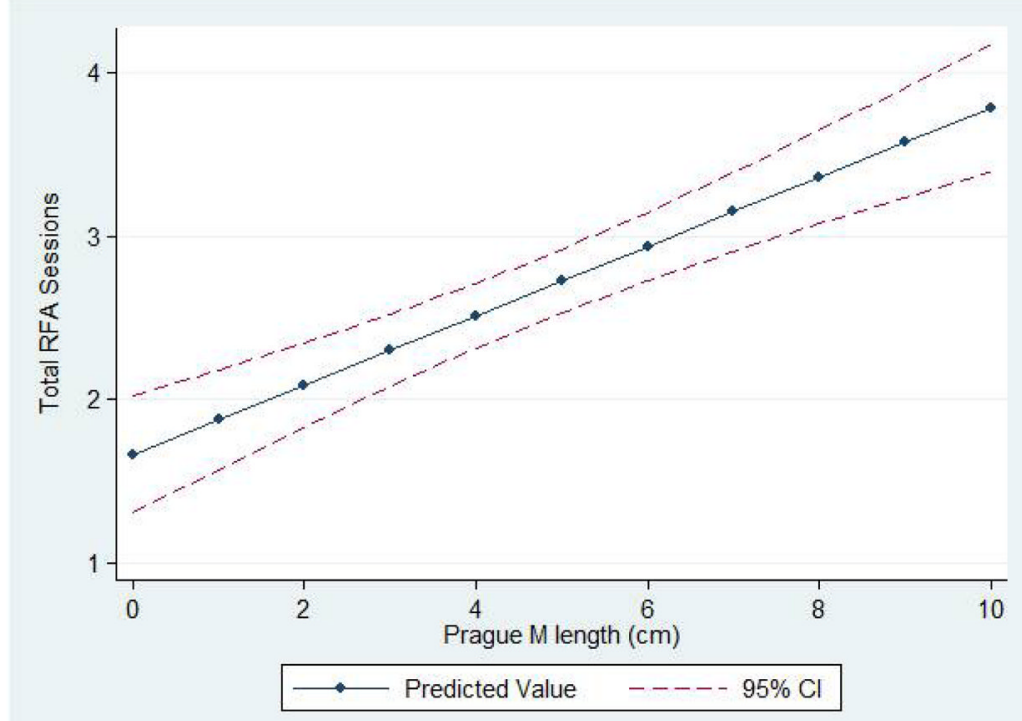

Figure 2. Predicted number of treatment sessions to achieve CEIM by Prague M length

* - Adjusted for age, gender, body mass index, NSAID use, presence of nodular disease, incomplete healing during treatment, medium or large hiatal hernia, and advanced histology (HGD, IMC). 
Table 2

Eradication rates, based on pre-treatment histological stage of disease

\begin{tabular}{|c|c|c|}
\hline & Per protocol, n (\%) & Intention-to-treat, $\mathbf{n}(\%)$ \\
\hline \multicolumn{3}{|l|}{ Any histology } \\
\hline $\mathbf{N}$ & 188 & 210 \\
\hline CED & $182(97)$ & $182(87)$ \\
\hline CEIM & $168(89)$ & $168(80)$ \\
\hline Total treatment sessions, mean (SD) & $3.2(1.7)$ & $3.3(1.8)$ \\
\hline RFA treatment sessions, mean (SD) & $2.7(1.5)$ & $2.8(1.7)$ \\
\hline \multicolumn{3}{|l|}{ Low-grade dysplasia } \\
\hline $\mathbf{N}$ & 41 & 44 \\
\hline CED & $41(100)$ & $41(93)$ \\
\hline CEIM & $38(93)$ & $38(86)$ \\
\hline Total treatment sessions, mean (SD) & $3.0(1.6)$ & $3.2(1.9)$ \\
\hline RFA treatment sessions, mean (SD) & $2.8(1.7)$ & $3.0(2.0)$ \\
\hline \multicolumn{3}{|l|}{ High-grade dysplasia } \\
\hline $\mathbf{N}$ & 118 & 135 \\
\hline CED & $114(97)$ & $114(84)$ \\
\hline CEIM & $106(90)$ & $106(79)$ \\
\hline Total treatment sessions, mean (SD) & $3.1(1.5)$ & $3.1(1.6)$ \\
\hline RFA treatment sessions, mean (SD) & $2.7(1.3)$ & $2.7(1.5)$ \\
\hline \multicolumn{3}{|l|}{ Intramucosal carcinoma } \\
\hline $\mathbf{N}$ & 29 & 31 \\
\hline CED & $27(93)$ & $27(87)$ \\
\hline CEIM & $24(83)$ & $24(77)$ \\
\hline Total treatment sessions, mean (SD) & $4.0(2.4)$ & $4.1(2.4)$ \\
\hline RFA treatment sessions, mean (SD) & $2.9(1.9)$ & $2.9(2.0)$ \\
\hline
\end{tabular}


Table 3

Comparison of subjects attaining complete eradication of intestinal metaplasia to those who did not

\begin{tabular}{|c|c|c|c|}
\hline \multirow[b]{2}{*}{ Characteristic } & \multicolumn{2}{|c|}{ Elimination of Intestinal Metaplasia } & \multirow[b]{2}{*}{ p-value } \\
\hline & Incomplete $(\mathbf{n = 2 0})$ & Complete $(\mathrm{n}=168)$ & \\
\hline Age, yrs (IQR) & $67.7(56.8,77.8)$ & $64.0(57.2,71.6)$ & 0.24 \\
\hline Male (n) & $60 \%(12)$ & $80 \%(134)$ & 0.045 \\
\hline Body mass index, $\mathrm{kg} / \mathrm{m}^{2}$ (IQR) & $31.6(27.2,35.6)$ & $29.3(25.8,33.5)$ & 0.24 \\
\hline \multicolumn{4}{|l|}{ Pre-treatment histology (n) } \\
\hline LGD & $15 \%(3)$ & $23 \%(38)$ & \\
\hline HGD & $60 \%(12)$ & $63 \%(106)$ & $0.20 *$ \\
\hline IMC & $25 \%(5)$ & $14 \%(24)$ & \\
\hline NSAID use (n) & $50 \%(10)$ & $51 \%(86)$ & 0.92 \\
\hline Medium or larger hiatus hernia (n) & $65 \%(13)$ & $56 \%(94)$ & 0.48 \\
\hline Length of BE, cm (IQR) & $5.5(3.5,8.5)$ & $4(1,6)$ & 0.03 \\
\hline Nodular BE (n) & $30 \%(6)$ & $29 \%(48)$ & 1.00 \\
\hline EMR during treatment (n) & $45 \%(9)$ & $35 \%(59)$ & 0.32 \\
\hline Incomplete healing between treatment sessions (n) & $45 \%(9)$ & $15 \%(26)$ & 0.004 \\
\hline Total treatment sessions (IQR) & $4(3,5)$ & $3(2,4)$ & 0.007 \\
\hline RFA treatment sessions (IQR) & $3(2,4)$ & $2(2,3)$ & 0.11 \\
\hline Treatment days (IQR) & $227(127,301)$ & $126(63,218)$ & 0.03 \\
\hline
\end{tabular}

p-value for trend 
Table 4

Comparison of subjects developing stricture to those who did not

\begin{tabular}{|c|c|c|c|}
\hline Characteristic & Stricture $(\mathbf{n}=\mathbf{2 0})$ & Stricture-Free $(n=224)$ & p-value \\
\hline Age, yrs (IQR) & $64.1(61.3,69.2)$ & $65.4(57.3,72.6)$ & 0.63 \\
\hline Male (n) & $65 \%(13)$ & $80 \%(179)$ & 0.15 \\
\hline Body mass index, $\mathrm{kg} / \mathrm{m}^{2}$ (IQR) & $29.0(26.9,35.4)$ & $29.0(25.8,33.0)$ & 0.53 \\
\hline History of prior stricture (n) & $15 \%(3)$ & $4 \%(9)$ & 0.06 \\
\hline History of prior erosive esophagitis (n) & $35 \%(7)$ & $12 \%(27)$ & 0.01 \\
\hline \multicolumn{4}{|l|}{ Pre-treatment histology (n) } \\
\hline LGD & $30 \%(6)$ & $21 \%(47)$ & \\
\hline HGD & $45 \%(9)$ & $64 \%(143)$ & 0.20 \\
\hline IMC & $25 \%(5)$ & $15 \%(34)$ & \\
\hline NSAID use (n) & $70 \%(14)$ & $45 \%(100)$ & 0.04 \\
\hline Medium or larger hiatus hernia (n) & $65 \%(13)$ & $58 \%(224)$ & 0.64 \\
\hline Intact Nissen fundoplication (n) & $15 \%(3)$ & $3 \%(7)$ & 0.04 \\
\hline Length of BE, cm (IQR) & $5(2,6.5)$ & $4(2,7)$ & 0.74 \\
\hline Nodular BE (n) & $25 \%(5)$ & $30 \%(68)$ & 0.80 \\
\hline EMR during treatment (n) & $20 \%(4)$ & $9 \%(21)$ & 0.62 \\
\hline Incomplete healing between treatment sessions (n) & $35 \%(7)$ & $17 \%(37)$ & 0.06 \\
\hline Total treatment sessions (IQR) & $3(2,5)$ & $3(2,4)$ & 0.09 \\
\hline RFA treatment sessions (IQR) & $3(1,4)$ & $2(1,3)$ & 0.36 \\
\hline Treatment days (IQR) & $154(98,262)$ & $123(61,228)$ & 0.16 \\
\hline
\end{tabular}

